\title{
Coupled Waveguides with MEMS Actuation for Continuously Tunable Optical Buffering
}

\author{
Peter Horak, Will Stewart, and Wei H. Loh \\ Optoelectronics Research Centre, University of Southampton, Southampton SO 17 1BJ, United Kingdom \\ e-mail: peh@orc.soton.ac.uk
}

\begin{abstract}
We propose a chip-based optical buffer that is continuously tunable by microelectromechanical actuation. The device consists of two identical semiconductor waveguides with a mechanically variable gap in-between, allowing for a change of group delay of over $100 \%$ and for extremely broadband operation.
\end{abstract}

Keywords: Optical buffer; Optical microelectromechanical devices; Integrated optics.

\section{INTRODUCTION}

The flexibility to store data in an optical format without the need for optical-electronic-optical conversion has a lot of potential for high speed optical communications and optical information processing, e.g. in packetswitched optical networks, and in optical processing on computer chips. Despite a range of efforts so far, however, a convenient and practical implementation of a tunable optical buffer still appears to be some distance away.

Fixed length optical delay lines are perhaps the most obvious approach [1], but they suffer from the inflexibility of having a fixed and predetermined data storage time in the resulting optical buffer. This limitation can be somewhat mitigated by using a delay line within a circulating loop, thus reducing the fixed restriction to delays of integer multiples of the single loop delay [1,2]. Further flexibility can be gained by switching between a range of different delay lines, but at the cost of increasingly bulky and complex devices.

Optical ring resonators are frequently employed for a compact solution [3], although such optical buffers are necessarily limited in operating bandwidth, due to their inherent trade-off between the delay time of the light spent circulating in the resonator and the resulting spectral bandwidth. Similarly, slow light buffers based on electromagnetically induced transparency (EIT) [4] provide large delays over a very narrow bandwidth only.

Here, we propose a different approach to achieve optical buffering, based on mechanically modifying the geometry of a dual-waveguide structure, which allows for a continuous change of the time delay by more than $100 \%[5]$.

\section{DEVICE DESIGN AND OPERATION}

A schematic of our proposed optical buffer is shown in Fig. 1. Two parallel identical silicon waveguides of width $w$ and height $h$ are mounted in air and are separated by a distance $d$. The separation can be varied continuously by mechanically moving one or both of the waveguides. This actuation can be achieved by standard MEMS techniques, e.g. electrostatic actuators [6]. Note that the pillars required for supporting the two waveguides as well as the electrodes required for the MEMS action are omitted for clarity in Fig. 1.

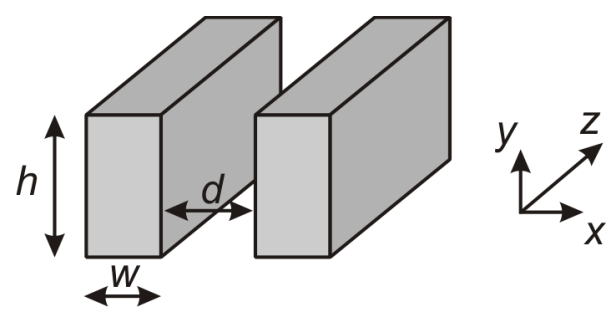

Figure 1. Schematic of the proposed dual-waveguide optical buffer.

For large separation $d$, each waveguide supports a single optical mode with quasi-TE polarization, i.e., with polarization predominantly in the $y$-direction. The modes of the dual-waveguide structure are simply given by the symmetric and antisymmetric (around the $x=0$ plane) superpositions of the individual modes. Moving the two waveguides together, the two individual modes become coupled through their evanescent fields, which modifies the dispersion properties and thus the group delay of the superposition modes. In the limit of small $d$, the antisymmetric mode reaches cutoff, whereas the symmetric mode converges towards the fundamental mode of the larger waveguide (width $2 w$, height $h$ ) formed by completely joining the two individual waveguides. In the following, we focus on this symmetric TE mode and investigate its properties numerically. The simulations are 
performed using a fully vectorial FEM solver (COMSOL Multiphysics $®$ ) and assuming a refractive index of silicon of 3.4 and waveguide dimensions of $h=300 \mathrm{~nm}, w=140 \mathrm{~nm}$, comparable to previously fabricated silicon waveguides [3].

(a)

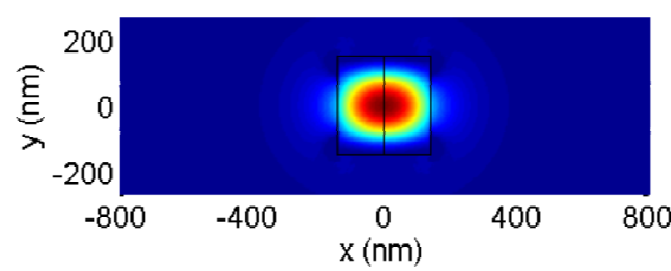

(b)

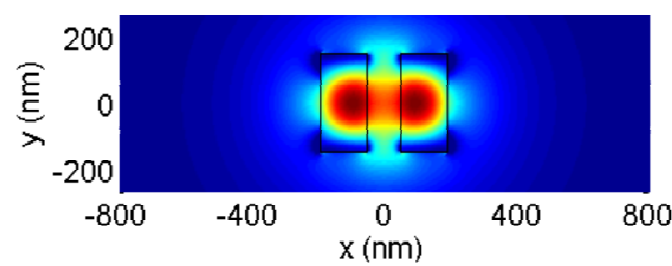

(c)

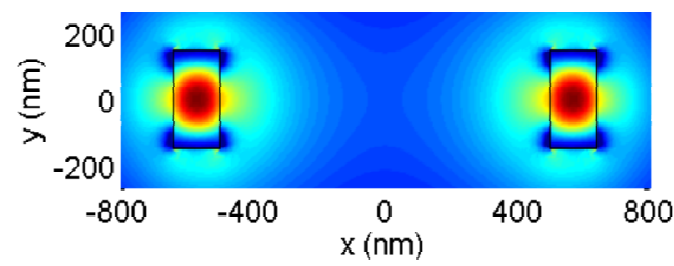

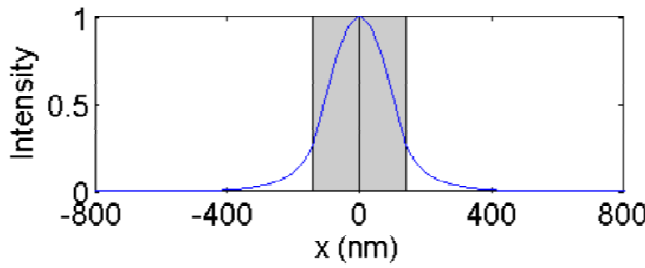
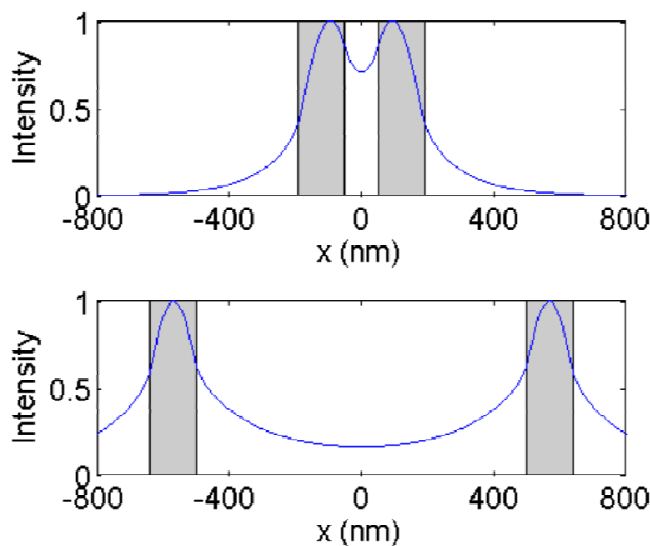

Figure 2. Symmetric TE mode functions of the dual waveguide structure with $h=300 \mathrm{~nm}, \mathrm{w}=140 \mathrm{~nm}$ for separations (a) $d=0 \mathrm{~nm}$, (b) $d=100 \mathrm{~nm}$, (c) $d=1000 \mathrm{~nm}$. Top row shows intensity contour plots, bottom row shows central $1 D$ cross sections of the normalized intensity.

For these parameters, the behaviour of the dual-waveguide symmetric TE mode is shown in Fig. 2, clearly demonstrating the transition from one tightly confined mode in the case of vanishing or small separation to a double-peaked mode with a significant fraction of power propagating in air for large separation. Consequently, the effective index of the mode, inversely proportional to the phase velocity, can be tuned continuously over a certain range between the refractive index of silicon $(n=3.4)$ and that of air $(n=1.0)$. This is shown more clearly in Fig. 3(a) which depicts the phase velocity versus $d$.

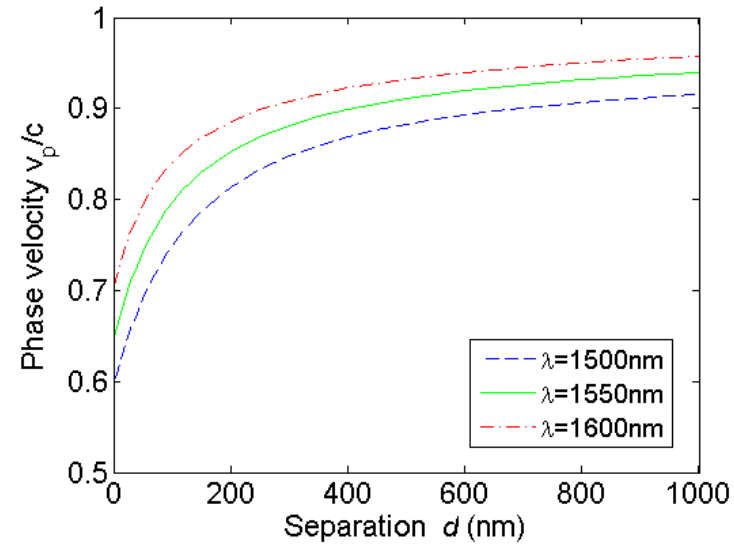

(a)

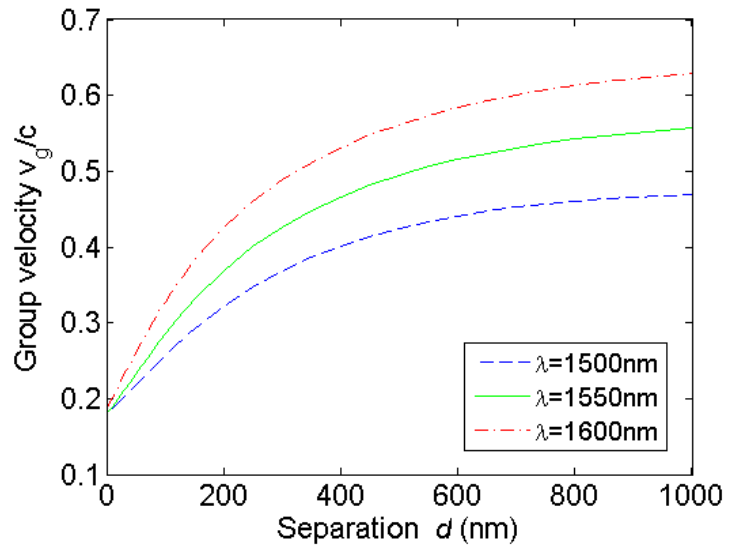

(b)

Figure 3. (a) Phase velocity and (b) group velocity of the device versus waveguide separation $d$. Parameters as in Fig. 2.

For the optical buffer functionality of the device, we are concerned with the behaviour and the tunability of the group velocity, which is inversely proportional to the time delay of optical pulses. The group velocity behaviour is shown in Fig. 3(b). We note that the group delay can be varied continuously through varying the 
waveguide separation $d$, by as much as a factor of three, with most of the change occurring within the first 500 nm of separation. With such a large controllable delay change, incorporating the device within a circulating loop would enable optical buffering for any arbitrary time. It is also worth emphasizing that the proposed buffer design is intrinsically broadband, in contrast to, in particular, resonator and EIT based optical buffers, with $>100 \%$ tunability over the entire wavelength range from $1500 \mathrm{~nm}$ to $1600 \mathrm{~nm}$. However, there is a significant variation of the exact time delay for a fixed waveguide separation within this wavelength range. This is related to the group velocity dispersion $D$ of the device, as shown more clearly in Fig. 4 as a function of the separation $d$. In fact, dispersion turns out to be not insignificant, $D \sim 10^{4} \mathrm{ps} / \mathrm{nm} / \mathrm{km}$. Note, however, that this is equivalent to a few $\mathrm{ps} / \mathrm{nm}$ for $1 \mathrm{~ns}$ of propagation time (approximately $10 \mathrm{~cm}$ distance) which is very small compared with resonator optical buffers.

Finally, we note that while the examples presented here are all based on TE-mode operation, similar performance can also be achieved with the symmetric TM mode ( $x$-polarization in Fig. 1) [5]. For TM operation, the optimum waveguide dimensions to achieve a threefold change in delay time would be slightly larger $(300 \mathrm{~nm}$ x $200 \mathrm{~nm}$ ) which may make it slightly easier to fabricate. On the other hand, the TM polarization leads to field discontinuities at the waveguide boundaries with strong field enhancement between the two waveguides, effectively operating like a slot waveguide [7]. The optical buffer operating in the TM polarization mode would therefore be more susceptible to scattering losses from surface roughness.

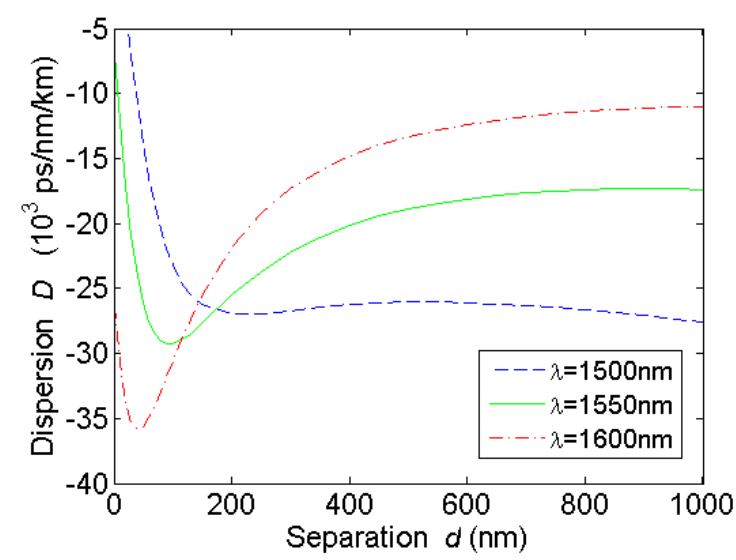

Figure 4. Group velocity dispersion D of the device versus waveguide separation $d$.

\section{CONCLUSIONS}

In conclusion, we have described the design and function of a dual-waveguide optical buffer that is continuously tunable in its propagation time delay through mechanically varying the separation between the two guides. The device would be capable of producing arbitrary time delays over the entire 1500-1600 nm wavelength range if used in a circulating loop. The proposed buffer can be implemented in either silicon or III-V semiconductor platforms, using existing MEMS technology for the required mechanical actuation. Several practical design issues will have to be addressed, such as efficient coupling into the symmetric TE mode - likely to be achieved by a waveguide taper and a subsequent $\mathrm{Y}$-junction - and maintaining low loss propagation at the contact points of the supporting structures with the optical waveguides - for which tapered designs have been shown to give losses as low as $0.02 \mathrm{~dB}$ per suspension bridge [8]

Although the analysis here has been with silicon waveguides, similar tunable delay lines could be implemented with optical fibres comprising twin cores with an adjustable separation between them [9]. With the index of the glass in fibres typically lower than for silicon, the relative changes achievable in the propagation time delay will be less; however, with fibres $\sim 100 \mathrm{~m}$ in length, tunable delays in the tens of ns would be feasible.

\section{ACKNOWLEDGEMENTS}

This work was partly supported by the UK Engineering and Physical Sciences Research Council via a Programme Grant on the 'Photonic Hyperhighway' project, EP/I01196X/1.

\section{REFERENCES}

[1] R. Langenhorst, M. Eiselt, W. Pieper, G. Großkopf, R. Ludwig, L. Kuller, E. Dietrich, and H. G. Weber: Fiber Loop Optical Buffer, J. Lightwave Technol., vol. 14, pp. 324-335, 1996. 
[2] H. Park, J. P. Mack, D. J. Blumenthal, and J. E. Bowers: An integrated recirculating optical buffer, Opt. Express, vol. 16, pp. 11124-11131, 2008.

[3] F. Xia, L. Sekaric, and Y. Vlasov: Ultracompact optical buffers on a silicon chip, Nature Photonics, vol. 1, pp. 65-71, 2007.

[4] J. B. Khurgin: Optical buffers based on slow light in electromagnetically induced transparent media and coupled resonant structures: a comparative analysis, J. Opt. Soc. Am. B, vol. 22, pp. 1062-1074, 2005.

[5] P. Horak, W. Stewart, and W. H. Loh: Continuously tunable optical buffer with a dual silicon waveguide design, Opt. Express, vol. 19, pp. 12456-12461, 2011.

[6] M. M. Lee and M. C. Wu: Tunable coupling regimes of silicon microdisk resonators using MEMS actuators, Opt. Express, vol. 14, pp. 4703-4712, 2006.

[7] V. R. Almeida, Q. Xu, C. A. Barrios, and M. Lipson: Guiding and confining light in void nanostructure, Opt. Lett., vol. 29, pp. 1209-1211, 2004.

[8] L. Martinez and M. Lipson: High confinement suspended micro-ring resonators in silicon-on-insulator, Opt. Express, vol. 14, pp. 6259-6263, 2006.

[9] Z. G. Lian, et al.: Optical fiber with dual cores suspended in air, in 37th European Conference on Optical Communication (ECOC) 2011, Geneva, Switzerland, September 2011, paper Mo.2.LeCervin.1 